\title{
A BOUNDED DIFFERENCE PROPERTY FOR CLASSES OF BANACH-VALUED FUNCTIONS
}

BY

\author{
WILBUR P. VEITH( ${ }^{1}$ )
}

ABSTRACT. Let $A(G, E)$ denote the set of functions $f$ from a Hausdorff topological group $G$ to a Banach space $E$ such that the range of $f$ is relatively compact in $E$ and $\phi \circ f$ is in $A(G, C)$ for each $\phi$ in the dual of $E$, where $A(G, C)$ is a translation-invariant $C^{*}$ algebra of bounded, continuous, complexvalued functions on $G$ with respect to the supremum norm and complex conjugation. $A(G, E)$ has the bounded difference property if whenever $F: G \rightarrow E$ is a bounded function such that $\Delta_{i} F(x)=F(t x)-F(x)$ is in $A(G, E)$ for each $t$ in $G$, then $F$ is also an element of $A(G, E)$. A condition on $A(G, C)$ and a condition on $E$ are given under which $A(G, E)$ has the bounded difference property. The condition on $A(G, C)$ is satisfied by both the class of almost periodic functions and the class of almost automorphic functions.

I. Introduction. The prototype of the results of this paper is the classical theorem of $\mathrm{H}$. Bohr [3] which states that if a complex-valued almost periodic function defined on the real line has a bounded primitive, then the primitive is itself almost periodic.

If $G$ is a Hausdorff topological group, R. Doss [5] showed that if $F$ is a bounded complex-valued function on $G$ and $\Delta_{t} F(x)=F(t x)-F(x)$ is almost periodic for each $t$ in $G$, then $F$ is almost periodic. This result generalizes Bohr's theorem.

Let $G$ be a Hausdorff topological group, $C$ the complex numbers, $E$ a Banach space, and $E^{*}$ the dual of $E . A(G, C)$ will denote a translation-invariant $C^{*}$ algebra with identity of bounded, continuous, complex-valued functions on $G$ with respect to the supremum norm and complex conjugation. $M(A)$ will denote the maximal ideal space for $A(G, C)$. A function $f: G \rightarrow E$ will be said to belong to $A(G, E)$ in case: (1) the range of $f$ is relatively compact in $E$, and (2) for each

Received by the editors May 15, 1972.

AMS (MOS) subject classifications (1970). Primary 46J10, 43A60; Secondary 46 E40.

Key words and phrases. Vector-valued almost periodic functions, vector-valued almost automorphic functions, bounded difference property, integration of almost periodic functions, Banach spaces with no copy of $c_{0}$.

(1) This research, which was prepared while the author was a university dissertation fellow, constitutes a portion of a doctoral dissertation written at The Ohio State University under the direction of Professors F. W. Carroll and J. A. Zilber. 
$\phi \in E^{*}$, the function $\phi \circ f$ belongs to $A(G, C)$. Our problem is to give conditions on $G, E$, and the algebra $A(G, C)$ so that the following bounded difference property holds:

Definition 1.1. The class of functions $A(G, E)$ has the bounded difference property (BDP) if whenever $F: G \rightarrow E$ is a bounded function such that $\Delta_{t} F(x)=$ $F(t x)-F(x)$ is in $A(G, E)$ for each $t$ in $G$, then $F$ is also an element of $A(G, E)$.

Since (BDP) fails to hold even for the almost periodic functions from the reals into $c$ (the space of convergent real sequences) [1, p. 53], but does hold in case $E$ is reflexive [10], it is clear that additional hypotheses will be needed.

When $G$ is the real line, $A(G, C)$ has (BDP) when $A$ is either the almost periodic or almost automorphic functions [2]. However, when $A$ is the set of functions which have a limit at infinity, $A(G, C)$ does not have the bounded difference property.

We shall show that $G$ operates on $M(A)$. In this way, we are able to define a classification of algebras $A(G, C)$ which distinguishes the almost automorphic and almost periodic functions from the functions which have a limit at infinity.

Definition 1.2. If the orbit of each element of $M(A)$ under the action of $G$ is dense in $M$, we shall say that $A(G, C)$ has the orbit property (0).

We can now state our principal result.

Theorem 1.3. If $A(G, C)$ bas the orbit property and if $E$ contains no subspace isomorphic to $c$, then $A(G, E)$ bas (BDP).

We remark that the first hypothesis concerns only $A(G, C)$, while the second hypothesis refers only to $E$. Examples of algebras $A(G, C)$ which satisfy this first hypothesis are the almost periodic functions and the almost automorphic functions.

The last section contains further results on the orbit property.

II. The maximal ideal space of $A(G, C)$. Let $M(A)$ denote the set of all algebra homomorphisms of $A(G, C)$ onto $C$. We define a function $\gamma$ from $G$ into $M$ by the formula $\gamma(g)(f)=f(g)$ for each $f \in A(G, C)$. With each function $f$ in $A(G, C)$ we associate a complex-valued function $\hat{f}$ on $M(A)$ by the rule $\hat{f}(\xi)=$ $\xi(f)$ for $\xi \in M(A)$.

$M(A)$ is given the weakest topology such that $\hat{f}$ is continuous on $M(A)$ for each $f$ in $A(G, C)$. A special case of a theorem proved in Loomis [7, p. 88] is that $\hat{:} A(G, C) \rightarrow C(M(A))$ is an isometry of $A(G, C)$ onto $C(M(A))$. The function $\gamma: G \rightarrow M(A)$ is continuous, and so $\gamma(G)$ must be dense in $M(A)$. Otherwise there would be a nonzero continuous function $\hat{f}$ on $M(A)$ whose correspondent $f$ on $G$ is identically zero, in contradiction to the result that ${ }^{{ }^{\wedge}}$ is an isometry onto $C(M(A))$. 
Theorem 2.1. Suppose $f$ is a function on $G$ with values in a Banach space $E$. Then $f$ is in $A(G, E)$ if and only if there is a continuous function $\hat{f}: M(A) \rightarrow E$ such that $\hat{f}(\gamma(x))=f(x)$ for each $x$ in $G$.

Proof. Suppose $f$ is in $A(G, E)$. Then $\phi \circ f$ is in $A(G, C)$ for each $\phi \in E^{*}$, and the range of $f$ is relatively compact in $E$. If $x$ and $y$ are two elements in $G$ such that $\gamma(x)$ equals $\gamma(y)$, then $\phi(f(x))$ equals $\phi(f(y))$ for each $\phi$ in $E^{*}$. By the Hahn-Banach theorem, $f(x)$ equals $f(y)$.

This shows that $f$ can be considered as a function on $\gamma(G)$. Call this new function $\hat{f}$. Let $t_{\alpha}, t_{a}^{\prime}$ be two nets in $\gamma(G)$ converging to $\xi$ in $M(A) . \hat{f}\left(t_{\alpha}\right)$ and $\hat{f}\left(t_{a}^{\prime}\right)$ are two nets in the relatively compact range of $f$, so each net has at least one cluster point. Since for each $\phi$ in $E^{*}, \phi \circ /$ is in $A(G, C)$, it has a continuous extension $(\phi \circ f)^{\wedge}$ to all of $M(A)$, and the equalities:

$$
\lim _{a} \phi\left(\hat{f}\left(t_{a}\right)\right)=(\phi \circ f)^{\wedge}(\xi) \text { and } \lim _{a^{\prime}} \phi\left(\hat{f}\left(t_{a}^{\prime}\right)\right)=(\phi \circ f)^{\wedge}(\xi)
$$

show that the two nets $\hat{f}\left(t_{\alpha}\right)$ and $\hat{f}\left(t_{a}^{\prime}\right)$ each have only one cluster point, hence are convergent. Moreover, the above equalities combined with the Hahn-Banach theorem show that the two nets $\hat{f}\left(t_{a}\right)$ and $\hat{f}\left(t_{a}^{\prime}\right)$ converge to the same limit.

Hence $\hat{f}$ is continuously extensible to $M(A)$.

Conversely, suppose there is a continuous function $\hat{f}: M(A) \rightarrow E$ such that $\hat{f}(\gamma(x))=f(x)$ for each $x$ in $G$. The range of $f$ is contained in the compact range of $\hat{f}$, hence is relatively compact in $E$. Also, for each $\phi$ in $E^{*}$ and $x$ in $G$, the equality $\phi \circ f(x)=\phi \circ \hat{f}(\gamma(x))$, shows that $\phi \circ f$ is in $A(G, C)$, due to the isometry of $C(M(A))$ and $A(G, C)$.

Since $\gamma(G)$ is dense in $M(A)$, when $\hat{F}$ is a function on $\gamma(G)$ with values in a Banach space $E$, we can determine when $\hat{F}$ is continuously extensible to $M(A)$ by using the following definition:

Definition 2.2. Suppose $\hat{F}$ is a function defined on $\gamma(G)$ with values in $E$. For $\xi$ in $M(A)$, we define the oscillation of $\hat{F}$ at $\xi$ as follows: osc $(\xi, \hat{F})=$ $\sup \left\{b \in R\right.$ : for each $\epsilon>0$ and each neighborhood $V$ of $\xi$, there exists $\xi^{\prime}, \xi^{\prime \prime}$ in $V \cap \gamma(G)$ such that $\left.\left\|\hat{F}\left(\xi^{\prime}\right)-\hat{F}\left(\xi^{\prime \prime}\right)\right\|>b-\epsilon\right\}$.

We shall say $\hat{F}$ is continuous at a point $\xi$ in $M(A)$ if and only if osc $(\xi, \hat{F})$ is zero. Moreover, the set of all $\xi$ with $\operatorname{osc}(\xi, \hat{F}) \geq 1 / n$ is closed.

III. The orbit property. In this section, we shall develop a condition on $A(G, C)$ which we shall call the orbit property.

Lemma 3.1. Suppose $\left\{\gamma\left(t_{a}\right)\right\}_{a \in \Lambda}$ is a net in $\gamma(G)$ converging to $\xi$ in $M(A)$, and that $b$ is an element of $G$. Then $\gamma\left(b t_{a}\right)$ is a convergent net in $M$.

Proof. In order for $\left\{y\left(b t_{a}\right)\right\}_{a \in \mathbf{A}}$ to be convergent in $M(A)$, it is necessary and sufficient that $\hat{f}\left(y\left(b t_{a}\right)\right)$ converges for each $f$ in $A(G, C)$, where $\hat{f}$ is the continuous 
function on $M(A)$ associated with $f$. This last statement also means $f_{b}\left(t_{a}\right)=$ $f\left(b t_{a}\right)$ converges for each $f$ in $A(G, C)$. Since $A(G, C)$ is translation invariant, $f_{b}$ is in $A(G, C)$ for each $b \in G$ and $f$ in $A(G, C)$. Thus, $f_{b}\left(t_{a}\right)$ converges to $\hat{f}_{b}(\xi)$, and it follows that $\left\{\gamma\left(b t_{a}\right)\right\}_{a \in \Lambda}$ converges in $M(A)$ whenever $\left\{y\left(t_{a}\right)\right\}_{a \in \Lambda}$ converges in $M(A)$.

Moreover, if $\left\{\gamma\left(t_{\beta}\right)\right\}_{\beta \in \Lambda}$, is another net in $\gamma(G)$ converging to $\xi$ in $M(A)$, then $\hat{f}_{b}\left(t_{\beta}\right)$ also converges to $\hat{f}_{b}(\xi)$, for $f \in A(G, C)$ and $b$ in $G$. This shows that the element $b \xi$ of $M(A)$ is well defined by the following definition:

Definition 3.2. If $\xi$ is in $M(A)$ and $b$ is in $G$, the element $b \xi$ of $M(A)$ is defined by

$$
b \xi=\lim _{a}\left\{\gamma\left(b t_{a}\right)\right\}_{a \in \Delta} \text {, }
$$

where $\left\{t_{a}\right\}_{a \in \Lambda}$ is any net in $G$ such that $\gamma\left(t_{\alpha}\right)$ converges to $\xi$ in $M(A)$.

In this way, $G$ operates on $M(A)$ by $b$ in $G$ carrying $\xi$ to $b \xi$. We denote the orbit of $\xi$ by orb $(\xi)$, where orb $(\xi)$ is the set $\{b \xi: b \in G\}$.

In [4], Carroll showed that a bounded function $f: G \rightarrow C$, each of whose left differences $\Delta_{t} f$ is almost periodic, can be considered as a function on $\gamma(G)$ in $M(A)$, the almost periodic compactification of $G$. Here $A$ denotes the almost periodic functions. This proof holds as well for any algebra $A(G, C)$ of the type we are discussing. In fact, if $f: G \rightarrow E$ is a bounded function each of whose left differences is in $A(G, E), E$ a Banach space, then $f$ can be considered as a function $\hat{f}$ on $\gamma(G)$. This is since $\phi \circ f$ satisfies the hypotheses of the Carroll lemma for each $\phi$ in $E^{*}$, so if $\gamma(x)$ equals $\gamma(y), \phi \circ f(x)$ equals $\phi \circ f(y)$ for each $\phi$ in $E^{*}$. By the Hahn-Banach theorem, $f(x)$ equals $f(y)$.

Thus, if $f: G \rightarrow E$ is a bounded function and $\Delta_{t} f$ is in $A(G, E)$ for each $t$ in $G$, then there is a function $\hat{f}: \gamma(G) \rightarrow E$ such that $\hat{f}(\gamma(x))$ equals $f(x)$ for each $x$ in $G$.

To show that $A(G, E)$ has the bounded difference property, it is enough to show that for such a function $f, \operatorname{osc}(\xi, \hat{f})$ is zero for each $\xi$ in $M(A)$.

Our analysis will be based primarily on the following theorem:

Theorem 3.3. Suppose $f: G \rightarrow E$ is a bounded function eacb of whose left difference is in $A(G, E)$. If $\xi$ is an element of $M(A)$ sucb that orb $(\xi)$ is dense in $M(A)$, and if there exists a point $\eta$ of $M(A) \operatorname{sucb}$ tbat osc $(\eta, \hat{f})$ is zero, then $\operatorname{osc}(\xi, \hat{f})$ is zero.

Proof. Choose $\epsilon>0$ arbitrarily. $O_{\epsilon}=\left\{\eta^{\prime} \in M(A): \operatorname{osc}\left(\eta^{\prime}, \hat{f}\right)<\epsilon\right\}$ is a nonempty open subset of $M(A)$. Choose $b$ in $G$ such that $b \xi$ is in the intersection of $O_{\epsilon}$ and orb $(\xi)$. We shall show that $\xi$ is in $O_{\epsilon}$. Let $t_{j}, t_{j}^{\prime}$ be two nets in $G$ such that $\gamma\left(t_{j}\right)$ and $\gamma\left(t_{j}^{\prime}\right)$ converge to $\xi$ in the topology of $M(A)$. In the inequality: 


$$
\begin{aligned}
& \left\|\hat{f}\left(\gamma\left(t_{j}\right)\right)-\hat{f}\left(\gamma\left(t_{j}^{\prime}\right)\right)\right\| \\
& \quad=\left\|\hat{f}\left(\gamma\left(t_{j}\right)\right)-\hat{f}\left(\gamma\left(b t_{j}\right)\right)+\hat{f}\left(\gamma\left(b t_{j}\right)\right)-\hat{f}\left(\gamma\left(b t_{j}^{\prime}\right)\right)+\hat{f}\left(\gamma\left(b t_{j}^{\prime}\right)\right)-\hat{f}\left(\gamma\left(t_{j}^{\prime}\right)\right)\right\| \\
& \quad \leq\left\|\Delta_{b} f\left(t_{j}\right)-\Delta_{b} f\left(t_{j}^{\prime}\right)\right\|+\left\|\hat{f}\left(\gamma\left(b t_{j}\right)\right)-\hat{f}\left(\gamma\left(b t_{j}^{\prime}\right)\right)\right\|
\end{aligned}
$$

the first term can be made arbitrarily small as $t_{j}, t_{j}^{\prime}$ approach $\xi$, by the fact that $\Delta_{b} f$ is in $A(G, E)$, hence is continuously extensible from $\gamma(G)$ to $M(A)$ by Theorem 2.1. The second term is eventually less than $\epsilon$ because $b \xi$ is in $O_{\epsilon}$. Thus, $\xi$ is in $O_{\epsilon}$. Since $\epsilon$ is arbitrary, osc $(\xi, \hat{f})$ is zero.

Because of this last result, we make the following definition:

Definition 3.4. We say $A(G, C)$ has the orbit property if orb $(\xi)$ is dense in $M(A)$ for each $\xi$ in $M(A)$.

IV. The proof of Theorem 1.3. In this section we shall prove that $A(G, E)$ has (BDP) whenever $A(G, C)$ has property $(O)$ and $E$ contains no subspace isomorphic to $c$. In view of Theorem 3.3, it is sufficient to prove:

Theorem 4.1. If $F: G \rightarrow E$ is bounded and $\Delta_{t} f$ is in $A(G, E)$ for eacb $t$ in $G$, and if $E$ contains no subspace isomorpbic to $c$, then $\hat{F}$ is continuous at $\mathcal{\gamma}(e)$, where $e$ is the identity of $G$.

Before presenting the proof of Theorem 4.1, we state a lemma due to Peĺczyński [8].

Lemma 4.2. Let $E$ be a Banach space. Suppose there is a divergent series $\Sigma X_{k}$ in $E$ such that all finite sums of its terms are uniformly bounded, $\left\|\Sigma X_{k_{i}}\right\| \leq$ $A<\infty$. Then $E$ contains a subspace isomorpbic to $c$.

Proof of Theorem 4.1. We proceed by contradiction. Suppose osc $(\mathcal{Z}(e), \hat{F})$ is not zero. We shall show that there is a divergent series in $E$ all finite sums of whose terms are uniformly bounded. This is contrary to Lemma 4.2, and will complete the proof.

Without loss of generality, we may assume $F(e)$ is zero. Since osc $(\mathcal{Z}(e), \hat{F})$ is not zero, we can find a real number $a$ greater than zero and a sequence $\left\{\gamma\left(t_{n}\right)\right\}$ in $\gamma(G)$ such that $\left\|\hat{F}\left(\gamma\left(t_{n}\right)\right)\right\| \geq a>0$ for each $n \in N$, and $\| \Delta_{\sigma} F\left(t_{n}\right)-$ $\Delta_{\sigma} F(e) \|<2^{-n}$, for $\sigma$ any product of elements of a subset of $\left\{t_{1}, t_{2}, \ldots, t_{n-1}\right\}$.

We shall now show that the divergent series $\Sigma \hat{F}\left(\gamma\left(t_{n}\right)\right)$ has a bound of $K+1$ for all finite sums of its terms, where $K$ is the bound for $F$.

Let $\tau_{1}, \tau_{2}, \ldots, \tau_{m}$ be any finite set of terms of the sequence $\left\{t_{n}\right\}$. We use the identity:

$$
F\left(\prod_{i=1}^{m} r_{i}\right)-\sum_{i=1}^{m} F\left(r_{i}\right)=\sum_{n=1}^{m-1}\left\{\Delta_{\sigma_{n}} F\left(r_{n+1}\right)-\Delta_{\sigma_{n}} F(e)\right\},
$$


where $\sigma_{n}=\tau_{1}, \tau_{2}, \ldots, \tau_{n}$, to show:

$$
\left\|\sum_{i=1}^{m} \hat{F}\left(\gamma\left(r_{i}\right)\right)\right\|=\left\|\sum_{i=1}^{m} F\left(r_{i}\right)\right\| \leq\left\|F\left(\prod_{i=1}^{m} r_{i}\right)\right\|+1 \leq K+1 .
$$

V. Related results. In this section, we shall apply the result of $\$ 4$ to almost periodic and almost automorphic functions and present some related results and comments.

Definition 5.1. Let $G$ be a topological group and let $E$ be a Banach space. $F: G \rightarrow E$ is an almost periodic function if it is a bounded continuous function and $\left\{f_{t}: f_{t}(x)=f(t x)\right\}$ is a relatively compact set in the space of bounded continuous functions from $G$ to $E$ with supremum norm.

Definition 5.2. Let $G$ be a topological group and let $E$ be a Banach space. We shall say that a function $f: G \rightarrow E$ is almost automorphic if it is continuous, and if each net $\left\{X_{a^{\prime}}\right\}_{a^{\prime} \in \Lambda^{\prime}}$ in $G$ contains a subnet $\left\{X_{a}\right\}_{a \in \Lambda}$ such that $\lim _{a \in \Lambda} \lim _{\beta \in \Lambda} f\left(\dot{X}_{\alpha}^{-1} X_{\beta} t\right)=f(t)$ holds for each $t$ in $G$.

Clearly the almost periodic functions are also almost automorphic.

Theorem 5.3. Let $A(G, C)$ be the set of almost automorphic functions from $G$ to $C$. Then $A(G, C)$ bas the orbit property.

Proof. Let $\xi$ be an arbitrary element of $M(A)$. If $e$ denotes the identity of $G$, it is easy to see that $\gamma(G)$ is contained in the closure of orb $(\xi)$ if $\gamma(e)$ is in the closure of $\operatorname{orb}(\xi)$. Since $\gamma(G)$ is dense in $M(A)$, it is sufficient, for the proof of this theorem, to show that $\gamma(e)$ is in the closure of orb $(\xi)$.

Let $f_{1}, f_{2}, \cdots, f_{n}$ be elements of $A(G, C)$, and let $\epsilon$ be an arbitrary positive number. A typical basic neighborhood of $\gamma(e)$ in $M(A)$ is:

$$
U=\left\{\eta \in M(A):\left|\hat{f}_{i}(\eta)-f_{i}(e)\right|<\epsilon, i=1,2, \ldots, n\right\},
$$

where $\hat{f}_{i}$ denotes the continuous function on $M(A)$ associated with $f_{i}$. Take any net $\alpha=\left\{t_{a}\right\}_{a \in \Lambda}$ of elements of $G$ such that $\gamma\left(t_{\alpha}\right)$ converges to $\xi$ in the topology of $M(A)$. Without loss of generality, we may assume $\lim _{\alpha \in \Lambda} \lim _{\beta \in \Lambda} f_{i}\left(t_{a}^{-1} t_{\beta} x\right)=$ $f_{i}(x)$ holds for each $x$ in $G$ and $i \in\{1,2, \cdots, n\}$. In particular, $\hat{f}_{i}\left(t_{\beta}^{-1} \xi\right)$ converges to $f_{i}(e)$ for $1 \leq i \leq n$. Choose $\beta_{0}$ so that $\beta$ finer than $\beta_{0}$ implies: $\left|\hat{f}_{i}\left(t_{\beta}^{-1} \xi\right)-f_{i}(e)\right|<\epsilon$ for $1 \leq i \leq n$. Then, taking $t=t_{\beta_{0}}^{-1}, t \xi$ is in $U$, so orb $(\xi)$ intersects $U$. Since $U$ was an arbitrary basic neighborhood of $\gamma(e), \gamma(e)$ is in the closure of orb $(\xi)$.

The proof of Theorem 5.3 in the case of almost periodic functions is similar.

Theorem 5.4. Let $R$ be the real line. Suppose $A(R, C)$ bas the orbit property. Then $A(R, C)$ contains no nonconstant functions which bave a limit at positive infinity. 
Proof. Suppose there exists a function $f$ in $A(R, C)$ which is continuous at $+\infty$, and $f$ is not a constant function. Without loss of generality, we may assume $f(0)$ is one and $f(+\infty)$ equals zero. Define $U$ by: $U=\{\xi \in M(A):|\hat{f}(\xi)-f(0)|<1 / 2\}$. $U$ is a neighborhood of $\gamma(0)$ in $M(A)$. Consider the sequence $N$. The corresponding sequence $\{y(n)\}_{n=1}^{\infty}$ in $\gamma(R)$ is a sequence in the compact set $M(A)$, hence has a convergent subnet $\left\{t_{\alpha}\right\}$ converging to some element $\xi$ in $M(A)$. We shall show that the orbit of $\xi$ is not dense by showing its orbit to be outside of $U$. For $t$ in $R$, the equation:

$$
\hat{f}(t+\xi)=\lim _{a} \hat{f}\left(t+t_{\alpha}\right)=\lim _{n \rightarrow \infty} f(t+n)=0,
$$

shows orb $(\xi)$ is in the complement of $U$.

In particular, consider $C_{c}(R)$, the set of continuous functions on $R$ which have a limit at infinity. This algebra of functions does not have the orbit property. Furthermore, this algebra does not have (BDP), for the function on $R$ with values in $C$ defined by: $F(x)=\sin \left(|x|^{1 / 2}\right)$ is a bounded function each of whose differences is in $C_{c}(R)$, but is not itself in $C_{c}(R)$.

In view of Theorem 5.3 and Theorem 4.1, whenever $A(G, E)$ is either the almost automorphic or almost periodic functions with values in a Banach space $E$, then $A(G, E)$ has (BDP) if $E$ contains no subspace isomorphic to $c$. The next example shows that this latter condition is also necessary for these two classes of functions to have (BDP) for an arbitrary group $G$.

Example 5.5. Let $Q$ be the rational numbers, and let $c$ be the space of convergent real sequences with supremum norm. Define $f: Q \rightarrow c$ by $f=\left\{f_{n}\right\}$, and $f_{n}(x)=\sin (2 \pi n ! x)$ for each $x$ in $Q$. Then, for each $x$ in $Q$, there exists $J(x)$ so that $j \geq J(x)$ implies $f_{j}(x)=0$. Thus, $f$ is clearly a function into $c$; in fact, at each point, $f$ defines a zero sequence.

Let $b$ be an element of $Q$, and write $b=p / q$, where $b$ is in this way written in lowest terms. (Note that $J(b)$ is less than or equal to $q$.) Since the equality:

$$
f(x+b)-f(x)=\left\{f_{1}(x+b)-f_{1}(x), \ldots, f_{J(b)}(x+b)-f_{J(b)}(x), 0,0, \ldots\right\}
$$

holds for each $b, \Delta_{b} f$ is continuous for each $b$ in $Q$. In fact, $\Delta_{b} f$ is almost periodic, for only finitely many of the almost periodic projections of $\Delta_{b} f$ are nonzero, and finitely many almost periodic functions are equi-almost periodic.

We shall now show that $f$ is not almost automorphic by showing that it is not even continuous at zero. Note that $f(0)$ is the sequence which is zero at each index. Consider the sequence $X_{m}=1 / 4 m$ !. Since we have:

$$
\left\|f\left(X_{m}\right)\right\| \geq f_{m}\left(X_{m}\right)=\sin ((2 \pi m !) / 4 m !)=1,
$$

$X_{m}$ converges to zero, but $f\left(X_{m}\right)$ does not converge to $f(0)$. 
In summary, the condition that $E$ contains no copy of $c$ is sufficient for $A(G, E)$ having (BDP) whenever $A(G, C)$ has the orbit property. It is a necessary condition if $A$ is the class of almost periodic or almost automorphic functions. The algebra $C_{c}(R)$ possesses neither the orbit property nor (BDP). It is possible that the condition that $E$ contains no copy of $c$ is equivalent to $A(G, E)$ having (BDP) if and only if $A(G, C)$ has the orbit property, when $G$ is an arbitrary group. Further investigation is required to determine whether this latter statement holds.

\section{REFERENCES}

1. L. Amerio and G. Prouse, Almost periodic functions and functional equations, Van Nostrand-Reinhold, New York, 1971. MR 43 \#819.

2. S. Bochner, $A$ new approach to almost periodicity, Proc. Nat. Acad. Sci. U. S. A. 48 (1962), 2039-2043. MR 26 \#2816.

3. H. Bohr, Almost periodic functions, Springer, Berlin, 1933.

4. F. W. Carroll, Difference properties for continuity and Riemann integrability on locally compact groups, Trans. Amer. Math. Soc. 102 (1962), 284-292. MR 24 \# A3438.

5. R. Doss, On bounded functions with almost periodic differences, Proc. Amer. Math. Soc. 12 (1961), 488-489. MR 23 \# A3424.

6. M. Kadec, The integration of almost periodic functions with values in a Banach space, Funkcional. Anal. i Priložen. 3 (1969), no. 3, 71-74. (Russian) MR 40 \# 4680.

7. L. H. Loomis, An introduction to abstract harmonic analysis, Van Nostrand, Princeton, N. J., 1953. MR 14, 883.

8. A. Pelczyński, On B-spaces containing subspaces isomorphic to the space $c_{0}$, Bull. Acad. Polon. Sci. Cl. III 5 (1957), 797-798. (Russian) MR 19, 565.

9. W. Veech, Almost automorphic functions on groups, Amer. J. Math. 87 (1965), 719-751. MR 32 \#4469.

10. W. P. Veith, Bounded Banach-valued functions with almost periodic differences, Bull. Un. Mat. Ital. (4) 4 (1971), 220-224. MR 45 \#9059.

DEPARTMENT OF MATHEMATICS, UNIVERSITY OF AKRON, AKRON, OHIO 44325 